\title{
How to Promote Patient Safety in Social Media: A Comparison between Messages in Social Media and Newspapers
}

\author{
Na Sun and Pei-Luen Patrick Rau \\ Institute of Human Factors and Ergonomics, Tsinghua University, Beijing 10084, China \\ \{sun-n10, rpl\} @mail.tsinghua. edu.cn
}

\begin{abstract}
The objective of this paper is to evaluate the features of patient-safety related messages in social media and provide suggestions for how to promote patient safety in social media. A hierarchical frame structure especially for patient safety messages was proposed for content analysis. 45 messages from Facebook and 45 reports from newspapers about patient safety were evaluated. The results indicated that messages in social media provided more comprehensive information about patient safety than newspapers. And social media paid more attention on individuals, including doctors, nurses and patients. Furthermore, social media messages provided more positive information than newspapers, such as treatment recommendations and program introductions.
\end{abstract}

Keywords: patient safety, social media, newspaper, content analysis.

\section{Introduction}

Patient safety is a new healthcare discipline that emphasizes the absence of preventable harm to patient during the process of health care (WHO). The impact of medical errors has been widely reported and discussed. Institute of Medicine of the National Academies reported that at least 44,000 Americans die each year as a result of medical errors (2000) [1]. More people die in a given year as a result of medical errors than from motor vehicle accidents $(43,458)$, breast cancer $(42,297)$, or AIDS $(16,516)$.

The improvement of patient safety requires the involvement of everyone, because anyone could become a patient sometime and the patients' awareness of patient safety issues is a major step to avoid medical errors. Traditional way to spread patient safety information is via newspapers. The reports in newspapers usually talked about patient safety issues with unexpected accidents or announcements made by organization. For instance, in the March 2010, the UK Medicines and Healthcare products Regulatory Agency (MHRA) released a Medical Device Alert in relation to all silicone gel filled breast implants manufactured by a French company called PIP. After the announcement, a large number of reports about PIP implant failure appeared in many newspapers. Some reports interviewed an unsuccessful implant surgery, and some reports presented the attitude of government and health institutions. 
Another way to spread patient safety information is via social media. Social media spreads information faster and more flexible than traditional media, especially among young people. Compared with newspapers, social media has less limitation of timesensitive, so the information can be more comprehensive and cover more topics, such as medical treatment tips and patient-safety related program introduction. Therefore, many patient-safety related organizations have set up their own public homepage on Facebook (the most popular social media), such as National Patient Safety Foundation and Canadian Patient Safety Institute. They use social network to introduce their organization development, to send announcements, and most importantly, to enhance people's awareness of patient safety issues. Social network has become an important way for patient safety education and promotion.

\section{Objective}

The initial objective of this paper was to find out the difference of patient-safety promotion in social media between China and America. However, this topic did not work out, because there were no public pages on patient safety in the most popular social network websites in China, including Renren.com, Kaixin.com, Weibo.com and T.qq.com. When using patient safety as the key word, only blogs sent by individual returned, and most of the messages had nothing to do with patient safety issues. Even though there were several organizations concentrating on health care promotion in China, none of them built public pages in social media.

Patient-safety promotion in social media is far from satisfactory in China. Similar situation can be observed in other developing countries. Therefore, suggestions on how to use social media to promote patient safety are needed especially for patient safety organizations. The objective of this study is to evaluate the features of patient safety messages in social media, i.e. Facebook, and therefore provide suggestions on patient-safety promotion in social media.

\section{$3 \quad$ Methodology}

\subsection{Hierarchical Content Analysis}

Over the past decades, the study of content analysis methodology has been rapidly expanding. A quantity of approaches has been proposed to measure the frame of content. All the approaches can be divided into five categories [15]. They are hermeneutic approach, linguistic approach, manual holistic approach, computer-assisted approach and deductive approach.

In this study, a mixture of hermeneutic approach and deductive approach was proposed. Firstly, content frames were divided into four dimensions according to previous literature [12]: problem definition, causal interpretation, moral evaluation, and treatment recommendation. Problem definition is the accident or potential risk mentioned in the messages. Causal interpretation identifies the reason that leads to the problem. Moral evaluation describes who should take the responsibility to solve the 
problem. And treatment recommendation refers to the suggestions provided for patients, physicians, hospitals, or government.

Secondly, a further exploration of key words in each dimension was conducted by hermeneutic approach. A coder read all the sample messages and identifies key words for each dimension. The qualitative assessment of framing depends on careful reading of all messages. For example, after reading a report about spa infection risks, the coder identified the key words as "infection" for problem definition; "lack of regulation for spa service" for causal interpretation; "patient, service-provider \& government" for moral evaluation; and "patient involvement" for treatment recommendation. Sometimes a message mentioned less than four dimensions of content frames.

After identify all the key words, the coder started to integrate the key words into systematic variables. The integration followed two principles: first, choose variables that cover as much as problems mentioned in the messages; second, variables are independent with each other, i.e. avoiding overlap among variables. The systematic variables formed the hierarchical frame structure. The hierarchical frame structure served as a codebook for content analysis.

At last, the coder reorganized the sample frame with hierarchical frame structure by adjusting key words into variables in each dimension. The frequency of variables revealed the feature of the sample messages, and therefore indicated the feature of the media. By using sample messages from both newspapers and social media, this paper compared the differences of messages between newspapers and social media and revealed the features of patient safety messages in social media.

\subsection{Sampling}

There are a number of public pages about patient safety on Facebook. Most of them are set up by non-profit organizations, communities and institutes, whereas some are interest groups, publishers and education groups. The most popular page is National Patient Safety Foundation, with 1,137 "likes" and 31 people "talking about this" in the past 7 days. The second popular page should be Canadian Patient Safety Institute, with 511 "likes" and 9 people "talking about this", followed by the page of Louise H. Batz Patient Safety Foundation with 258 "likes" and 4 "talking about this". The other pages attract relatively less attention, no more than 100 "likes". Thus these three most popular public pages were chosen as the source of sample messages. A total of 45 messages about patient safety were selected from their Facebook pages as the sample messages. Most of these messages contained a link to a new webpage representing a patient-safety related report. Only one message provided no further report but only some tips for patients.

Another set of sample messages were selected from an online database called Library.PressDisplay.com, which provides more than 800 online newspapers from around 70 countries all over the world. In order to avoid too many reports about the same accident, two time periods were chosen as sample time, which are Jan. 5 to Jan. 9 in 2012 and Feb. 18 to Feb. 22 in 2012. At last, 45 reports from different newspapers were selected as the second set of sample messages. 


\section{$4 \quad$ Results}

\subsection{Hierarchical Frame Structure}

By integrating all the key words abstracted from sample messages, the hierarchical frame structure was generated, as shown in table 1.

Table 1. Hierarchical Frame Structure

\begin{tabular}{ll}
\hline Problem Definition: & \\
\hline Medication error & Surgery error \\
Clinical nursing error & Nosocomial infection \\
Complication & Patient identification \\
Emergency treatment & Delay \\
Misdiagnose & Fatigue \\
Ambulatory care error & Fall \\
\hline Causal Interpretation: & \\
\hline Lack of training & Hospital management \\
Lack of resources & Lack of policy \\
Professional attitude & Bad facility \\
Finance & Hospital culture \\
Lack of knowledge & Cooperation failure \\
External distraction & \\
\hline Moral Evaluation: & \\
\hline Hospital & Government \\
Service-provider & Patient \\
\hline Treatment Recommendation: & \\
\hline Patient involvement & Training \\
Better medical system & Policy \\
Hospital culture & More privacy \\
Medical data releasing & Improving instruction \\
Technology support & Better cooperation \\
More researches & \\
\hline
\end{tabular}

According to the hierarchical frame structure, sample messages were divided into different problems, causes, responsibilities, and recommendations. The following parts explain the comparison results of Facebook and newspapers, which reveal the differences of patient safety messages in social media and in traditional newspapers.

\subsection{Types of Sample Messages}

Before systematically analyzing the frame of sample messages, a general description was put forward to evaluate what kind of information the messages provide. Four types of typical information were checked, which are statistic fact, practical accident, patient-safety related program or project and recommendation. The evaluation 
procedure came with questions such as "Is there any statistic fact in the message?" "Does the message mention any program or project to improve patient safety?" Sometimes a piece of message contained more than one type of information. The results are shown in Table 2 . The frequency refers to how many messages contain certain information and the percentage represents the percentage of messages that contain certain information.

Table 2. Information Types Analysis of Patient Safety Messages

\begin{tabular}{lcccc}
\hline \multirow{2}{*}{ Info Type } & \multicolumn{2}{c}{ Facebook } & \multicolumn{2}{c}{ PressDisplay } \\
\cline { 2 - 5 } & Freq. & $\%$ & Freq. & $\%$ \\
\hline statistic & 22 & 0.49 & 22 & 0.49 \\
recommendation & 22 & 0.49 & 18 & 0.40 \\
accident & 17 & 0.38 & 28 & 0.62 \\
program & 17 & 0.38 & 13 & 0.29 \\
\hline
\end{tabular}

The results indicated that four types of information appear averagely in social media, compared with newspapers. Social media messages contained more program introduction (38\%) than newspapers reports (29\%), so was the recommendation (49\% to $10 \%)$. But newspapers reported more accidents (62\%) than social media (38\%).

This can be easily explained by the difference of newspaper industry and social media. Newspaper companies are aimed at attracting more consumers by providing the most fresh and attractive news. But the public pages on Facebook are all set up by non-profit organization, whose goal is to spread patient safety information. As a result, newspapers preferred the latest accidents reports, and Facebook pages included as many programs and recommendations as accidents. Even though these messages may not be so attractive to readers, they are great progress in patient safety field and worth reading.

\subsection{Problem Definition}

45 sample messages from social media mentioned 10 problems; while 45 newspaper reports only included 8 problems. This result indicated that messages on Facebook covered more topics than newspapers. The percentage of each problem is shown in table 3. Among social media information, nosocomial infection (26.7\%) drew significantly more attention, followed by surgery error (6.7\%) and complication (6.7\%). As for newspapers, more than half of the messages focused on medication errors $(37.8 \%)$ and clinical nursing errors $(24.4 \%)$. This result is partly due to two events during the sample periods. The first one is an unsuccessful breast implant surgery accident, which is classified as medication error and surgery error. The other one is the publication of adverse medical events by Health Quality and Safety Commission, in which medication errors and clinical nursing errors are two common mishaps. As a result, the distribution of problems is unbalanced. Therefore, newspaper topics are relatively concentrated, and depend heavily on practical news of the sample period. 
Table 3. Content Frame Analysis: Problem Definition

\begin{tabular}{lcccc}
\hline \multirow{2}{*}{ Problem Definition } & \multicolumn{2}{c}{ Facebook } & \multicolumn{2}{c}{ PressDisplay } \\
\cline { 2 - 5 } & Freq. & $\%$ & Freq. & $\%$ \\
\hline nosocomial infection & 12 & 0.267 & 7 & 0.156 \\
surgery error & 3 & 0.067 & 9 & 0.200 \\
complication & 3 & 0.067 & 3 & 0.067 \\
medication error & 2 & 0.044 & 17 & 0.378 \\
clinical nursing error & 2 & 0.044 & 11 & 0.244 \\
emergency treatment delay & 2 & 0.044 & 0 & 0 \\
misdiagnose & 2 & 0.044 & 4 & 0.089 \\
fatigue & 2 & 0.044 & 0 & 0 \\
ambulatory care error & 1 & 0.022 & 0 & 0 \\
slip and fall & 1 & 0.022 & 4 & 0.089 \\
patient identification & 0 & 0 & 2 & 0.044 \\
\hline
\end{tabular}

\subsection{Causal Interpretation}

There were totally 12 interpretations, as shown in Table 4 . The most common cause was hospital management failure, followed by lack of medical resources in both media. But none of the accidents in newspapers were caused by hospital culture, which was the third frequent reason on Facebook (8.9\%). Facility (2.2\%) and finance issues $(2.2 \%)$ were two interpretations in newspapers, but they were not mentioned on Facebook. Facebook aims at improving the awareness and knowledge of patient safety issues, so it pays more attention on hospital and individuals. That is why 4 of 45 messages on Facebook ascribed problems to hospital culture.

Table 4. Content Frame Analysis: Causal Interpretation

\begin{tabular}{lcccc}
\hline \multirow{2}{*}{ Causal Interpretation } & \multicolumn{2}{c}{ Facebook } & \multicolumn{2}{c}{ PressDisplay } \\
\cline { 2 - 5 } & Freq. & $\%$ & Freq. & $\%$ \\
\hline hospital management failure & 11 & 0.244 & 7 & 0.156 \\
lack of resources & 5 & 0.111 & 5 & 0.111 \\
hospital culture & 4 & 0.089 & 0 & 0 \\
lack of policy & 3 & 0.067 & 2 & 0.044 \\
professional attitude & 2 & 0.044 & 1 & 0.022 \\
patient knowledge shortage & 1 & 0.022 & 2 & 0.044 \\
cooperation failure & 1 & 0.022 & 1 & 0.022 \\
lack of training & 1 & 0.022 & 4 & 0.089 \\
external distraction & 1 & 0.022 & 0 & 0 \\
bad facility & 0 & 0 & 1 & 0.022 \\
finance issues & 0 & 0 & 2 & 0.044 \\
\hline
\end{tabular}




\subsection{Moral Evaluation}

Moral evaluation identifies causal agents, which are taking responsibility for the accidents or risks, as in table 5. Hospital was the major causal agents in messages from both media. But patients took higher proportion of moral evaluation in Facebook messages $(11.1 \%)$ than newspaper reports $(4.4 \%)$, which means messages on Facebook focused more on individual responsibility regarding patient safety problems. Newspapers paid more attention on government responsibility for patient safety problems $(31.1 \%)$ than Facebook (15.6\%). This result indicated that Facebook focuses on individuals while newspapers focus on macro-environment.

Table 5. Content Frame Analysis: Moral Evaluation

\begin{tabular}{lcccc}
\hline \multirow{2}{*}{ Moral Evaluation } & \multicolumn{2}{c}{ Facebook } & \multicolumn{2}{c}{ PressDisplay } \\
\cline { 2 - 5 } & Freq. & $\%$ & Freq. & $\%$ \\
\hline hospital & 27 & 0.600 & 25 & 0.556 \\
service-provider & 9 & 0.200 & 9 & 0.200 \\
government & 7 & 0.156 & 14 & 0.311 \\
patient & 5 & 0.111 & 2 & 0.044 \\
\hline
\end{tabular}

\subsection{Treatment Recommendation}

Treatment recommendation refers to the suggestions for improving patient safety when accepting medical treatment. Table 6 shows 11 recommendations abstracted from the sample messages. The data revealed that messages from newspapers provided relatively less treatment recommendations than Facebook messages. The major recommendation in Facebook messages was patient involvement $(33.3 \%)$, following by training (15.6\%) and better medical system (15.6\%). Three of these treatment recommendations were considered as treatments for individuals: patient involvement, training and better cooperation. 20 of 45 Facebook messages provided treatment recommendation for individuals, but only 10 of 45 newspaper reports provided treatments for individuals.

Table 6. Content Frame Analysis: Treatment Recommendation

\begin{tabular}{lcccc}
\hline \multirow{2}{*}{ Treatment Recommendation } & \multicolumn{2}{c}{ Facebook } & \multicolumn{2}{c}{ PressDisplay } \\
\cline { 2 - 5 } & Freq. & $\%$ & Freq. & $\%$ \\
\hline patient involvement & 15 & 0.333 & 6 & 0.133 \\
training & 7 & 0.156 & 2 & 0.044 \\
better medical system & 7 & 0.156 & 6 & 0.133 \\
policy improvement & 4 & 0.089 & 2 & 0.044 \\
better technology & 4 & 0.089 & 0 & 0 \\
better cooperation & 4 & 0.089 & 1 & 0.022 \\
hospital culture improvement & 2 & 0.044 & 1 & 0.022 \\
\hline
\end{tabular}


Table 6. (continued)

\begin{tabular}{lllll}
\hline more privacy & 2 & 0.044 & 1 & 0.022 \\
information publicity & 1 & 0.022 & 3 & 0.067 \\
Better medical instruction & 1 & 0.022 & 1 & 0.022 \\
more researches & 1 & 0.022 & 2 & 0.044 \\
\hline
\end{tabular}

\section{Conclusion}

This study proposed a hierarchical content analysis method to evaluate the feature of patient safety messages in social media, i.e. Facebook. 45 messages from Facebook and 45 reports from newspapers were chosen as sample. The analysis results indicated the following findings.

1. Messages in social media provided more recommendations and program introductions than newspaper reports, while newspaper reports contained more practical accidents. Social media has fewer requirements for timeliness, so social media covers more kinds of information and provide more comprehensive knowledge of patient safety.

2. Facebook messages covered a larger scope of patient-safety related problems and risks. However, the problems in newspaper messages were more concentrated and usually related to recent accidents or policies. As a result, social media is more suitable to promote overall patient safety knowledge even though some of them seldom happens.

3. The most frequent causes were hospital management failure and lack of resources in both media. Hospital culture was a major cause in social media. But newspapers did not include culture issues, and instead newspaper reports talked more about finance and facility issues.

4. Facebook messages emphasized the role of the individual in improving patient safety more frequently than newspaper reports. Newspapers paid more attention on government responsibility than Facebook. Besides, both media indicated that hospital should take the major responsibility of patient safety problems.

5. Facebook messages provided more treatment recommendations than newspaper reports. And a large proportion of treatments were for individuals, including doctors, nurses, paramedic and patients.

\section{Discussion}

This paper aims to find out the differences of patient-safety related messages in social media and newspapers. The results revealed four features of patient safety messages in social media and provided suggestions on how to design effective messages that are suitable for social media.

Firstly, social media is a perfect platform for non-profit organizations to promote commonweal concept and knowledge, because anyone can send messages in social 
media for free. What's more, messages in social media can be spread rapidly through social network, and people with the same interest can be associated together by the same message. Organizations should make full use of social media advantages to make the messages more attractive and meaningful. For example, they can provide more colorful pictures and videos on their public pages, which seldom appear in newspapers. In addition, they can set up some activities among users about patient safety topic, such as patient safety knowledge contest, emergency treatment practice and medical environment survey.

Secondly, there are more program introductions and recommendations in social media than in newspapers. Thus positive messages are more appropriate to spread in social media than traditional media. Negative messages are usually astonishing and attract more readers, so traditional media is more likely to report negative information to attract consumers. In contrast, when spreading patient safety information in social media, organizations are aimed at providing comprehensive knowledge to readers, including both negative and positive messages. So there are more positive messages in social media than in newspapers. Besides, users who visit patient safety public pages in social media are concerned with patient safety issues, so the public pages should provide more inspiring and practical information for them. Even though some news is about tragic accident, further improving action or recommendation should be attached. This principle is not only for patient-safety promotion, but also for all the commonweal concept promotion, such as energy conservation, family planning and prevention of child maltreatment.

Another characteristic of social media messages is comprehensiveness. 45 sample messages in social media covered 10 patient safety problems, while 45 sample newspaper reports only mentioned 8 problems. Social media pay less attention on timeliness and readers' interest. Thus social media is a better platform to promote overall knowledge of patient safety. This is also true for other topics, such as non-smoking, healthy diet and animal protection. When spreading knowledge via social media, special attention should be paid on unusual knowledge to open users' minds. For example, National Patient Safety Foundation reported a program on ambulatory patient safety, which is a rare topic in traditional media.

The last characteristic of social media is individually oriented, which means messages in social media concentrate on individual concept and knowledge. For example, social media provides more treatment recommendations for individuals than newspapers. Traditional media usually has the function of public opinion and is able to influence the government, so it focuses more on macro-environment. In contrast, social media mainly aims to increase the awareness and knowledge of individual users, instead of impacting the macro-environment. As a result, more recommendations for patients, doctors and nurses should be provided in social media.

There are some limitations in this study. Firstly, the sample size is small, which partially due to the complexity of hermeneutic content analysis. Secondly, the procedure of content analysis is subjective, so individual differences may impact the results. In further studies, more objective methodology needs to be explored. And the analysis of social media messages should be extended to other topics. 


\section{References}

1. Kohn, L.T., Corrigan, J.M., Donaldson, M.S.: To Err is Human: Building a Safer Health System. National Academy Press, Washington, DC (2000)

2. Boyd, D.M.: Social network sites: definition, history, and scholarship. Journal of Computer-Mediated Communication 13, 210-230 (2008)

3. Lenhart, A.: Teens and Social Media: An Overview (2009)

4. Kaplan, A.M., Haenlein, M.: Users of the world, unite! The challenges and opportunities of Social Media. Business Horizons 53, 59-68 (2010)

5. Ehara, A.: Are long physician working hours harmful to patient safety. Pediatrics International 50, 175-178 (2008)

6. Ramanujam, R., Abrahamson, K., Anderson, J.G.: Influence of workplace demands on nurses' perception of patient safety. Nursing and Health Sciences 10, 144-150 (2008)

7. Chuang, S., Pan, C., Huang, C.: A system-oriented analysis model to enhance patient safety in healthcare organizations. Systems Engineering 12(3), 218-232 (2009)

8. Wachter, R.M.: The end of the beginning: Patient safety five years after "To Err Is Human". In: Health Affairs, pp. w4.534-w4.545 (2004)

9. Feng, X., Bobay, K., Weiss, M.: Patient safety culture in nursing: a dimensional concept analysis. Journal of Advanced Nursing 63(3), 310-319 (2008)

10. Colla, J.B., Bracken, A.C., Kinney, L.M., Weeks, W.B.: Measuring patient safety climate: a review of surveys. Quality \& Safety in Health Care 14, 364-366 (2005)

11. Semetko, H.A., Valkenburg, P.M.: Framing European politics: A content analysis of press and television news. Journal of Communication 50(2), 93-109 (2000)

12. Entman, R.M.: Framing: Toward clarification of a fractured paradigm. Journal of Communication 43(4), 51-58 (1993)

13. Akhavan-Majid, R., Ramaprasas, J.: Framing and ideology: A comparative analysis of U.S. and Chinese newspaper coverage of the Fourth United Nations Conference on Women and the NGO Forum. Mass Communication and Society 1(3-4), 131-152 (1998)

14. Graneheim, U.H., Lundman, B.: Qualitative content analysis in nursing research: concepts, procedures and measures to achieve trustworthiness. Nurse Education Today 24, 105-112 (2004)

15. Matthes, J., Kohring, M.: The content analysis of media frames: Toward improving reliability and validity. Journal of Communication 58, 258-279 (2008)

16. Segvic, I.: The framing of politics: A content analysis of three Croatian newspapers. The International Journal for Communication Studies 67(5), 469-488 (2005)

17. Hsieh, H.F., Shanno, S.E.: Three approaches to qualitative content analysis. Qualitative Health Research 15(9), 1277-1288 (2005) 